\title{
CAPACIDAD DE MEMORIA OPERATIVA \\ Y SEGUIMIENTO DE INSTRUCCIONES EN UNIVERSITARIOS
}

CAPACITY OF OPERATIONAL MEMORY AND FOLLOW-UP OF INSTRUCTIONS IN UNIVERSITY

Ismael Esquivel Gámez, Eric Efrain Solano Uscanga

Universidad Veracruzana, México / Correspondencia: iesquivel@uv.mx

\section{RESUMEN}

Este estudio analiza los niveles de asociación entre la capacidad de memoria operativa y el seguimiento de instrucciones, como elementos básicos para el desarrollo de competencias necesarias en ambientes educativos. Desde un enfoque cuantitativo, se usó un diseño de tipo correlacional, no experimental, transversal y prospectivo, en el cual participaron 33 universitarios de nuevo ingreso de la ciudad de Veracruz, México con 18.5 años de edad promedio, a los cuales se le aplicaron pruebas de alcance complejo para medir la capacidad de la memoria operativa. El seguimiento de instrucciones, se midió con cuatro pruebas que requerían de construir modelos con piezas Lego ${ }^{\circledR}$ a partir de la lectura de un procedimiento. Los resultados muestran una asociación positiva entre la habilidad para seguir instrucciones y la capacidad de la memoria operativa, de magnitud similar para sus dominios verbal y viso-espacial. Ambos tipos de prueba incorporados a una plataforma virtual de aprendizaje, puede proporcionar un ambiente válido y ecológico en la medición de ambas capacidades y su nivel de asociación, para la detección temprana de universitarios bajo riesgo académico. 
Palabras clave: Memoria operativa, seguimiento de instrucciones, habilidades cognitivas básicas y/o superiores

\section{ABSTRACT}

This study analyzes the levels of association between working memory capacity and following instructions, as basic elements for the development of required skills in educational environments. From a quantitative approach, a correlational, non-experimental, cross-sectional and prospective design was used, in which 33 undergraduates with an average age of 18.5 years from Veracruz, Mexico participated. The working memory capacity was measured with complex span tasks and the follow-up of instructions, with tests that required building models with Lego ${ }^{\circledR}$ pieces from the reading of procedures. The results show a positive association between the ability to follow instructions and the working memory capacity, in both, verbal and visuospatial domains. The tests incorporated into a virtual learning platform can provide a valid and ecological environment in the measurement of both capacities and their level of association, for the early detection of students under academic risk.

Keywords: Working memory, following instructions, basic and / or higher cognitive skills

\section{INTRODUCCIÓN}

En diversos ámbitos de la vida diaria de los estudiantes universitarios, parte importante del repertorio conductual es mantenido por el comportamiento verbal de otros, por ejemplo, a través de instrucciones (Donadelli y Strapasson, 2015). La habilidad para seguir instrucciones de manera exitosa es vital para el funcionamiento cognitivo efectivo y es estratégica ante distintas actividades cotidianas que han sido reconocidas como retadoras y 
propensas a errores (Gathercole y Alloway, 2007). De acuerdo con Ganier y de Vries (2016), la manera en que se presentan las instrucciones tiene un impacto directo en el procesamiento de la información en la memoria de trabajo. De este modo, el procesamiento de información contenida en las instrucciones requiere cierto nivel de esfuerzo cognitivo por parte del estudiante, y por lo tanto tiene un costo desde el punto de vista cognitivo. Por lo anterior, se ha desarrollado el presente estudio cuyo objetivo general es medir la capacidad de la memoria operativa y del seguimiento de instrucciones escritas, para determinar los niveles de asociación entre ambas variables de estudio. Para ello, inicialmente, se documentan las variables correspondientes, los estudios que han encontrado asociaciones entre ellas, particularidades en la medición de la memoria operativa, para luego dar paso a la descripción del método y la discusión de resultados y finalmente, presentar las conclusiones.

\section{MARCO CONCEPTUAL}

Entre las habilidades cognitivas superiores, la memoria de trabajo es considerada una función central (Miyake et al.,2000). La memoria de trabajo (MT) o memoria operativa (MO), se encarga del almacenar temporalmente y actualizar una pequeña cantidad de datos, que están accesibles para, mediante el uso de estrategias, posteriormente manipularlos en tiempo real (Jonides, Lacey y Nee, 2005). Para operaciones que implican comprender el lenguaje, procesar datos rápidamente y al resolver problemas, preservar los objetivos es de gran importancia (Baddeley, 2012; Etchepareborda y Abad-Mas, 2005; Flores y Ostrosky-Shejet, 2012).

El modelo de componentes múltiples de memoria operativa de Baddeley y Hitch (1974), reexaminado por Baddeley (2012) ha sido especialmente útil para su análisis. Este modelo propone que la MO está conformado por una 
central ejecutiva, que se divide en dos subsistemas: el bucle fonológico y la agenda visoespacial y, el búfer episódico. La central ejecutiva es una instancia de control de la atención para eventos relacionados con los contenidos dentro la memoria operativa así como para los que se encuentran fuera de ella (Jaroslawska, Gathercole, Logie y Holmes, 2016). El bucle fonológico es el depósito temporal de estímulos verbales, que maneja a su vez, dos subsistemas, un almacén de síntesis articulatoria y uno fonológico (Flores y Ostrosky-Shejet, 2012). La agenda viso-espacial, mantiene temporalmente información visual y espacial; la cual es usada para planear movimientos y está asociada a la aptitud espacial y tareas vinculadas (Etchepareborda y Abad-Mas, 2005). Por su parte, Arteaga y Pimienta (2006) proponen que está formada por componentes visuales, espaciales y cinéticos. Los primeros encargados de percibir "el qué"; los segundos de la ubicación y transmisión de "el dónde" y los últimos de apoyar el correcto movimiento. Por último, el búfer episódico es el encargado de mantener una relación entre los tres y la memoria de largo alcance; filtrar los estímulos conforme a su naturaleza y, aunque es un componente temporal, acceder a la memoria de largo plazo, sea para aprender o para recuperar (Saeed, 2011).

Por otro lado, cuando se procede a seguir una instrucción es necesario que se conserve con detalle el orden de las acciones requeridas por la misma, mientras que a la vez, se debe responder contingentemente a la retroalimentación generada por los efectos de las actividades que se llevan a cabo en tiempo real (Solano-Uscanga, de Vries y Edel, 2018). En su trabajo, Ganier y de Vries (2016) demostraron que el primer paso para llevar a cabo un procedimiento, se encuentra caracterizado por la necesidad de tener referencias sistemáticas de las instrucciones. En esta etapa, es probable que cada instrucción sea consultada al menos dos veces y el costo 
cognitivo asociado, trae como resultado la necesidad de un mayor tiempo para llevar a cabo el procedimiento que en los intentos subsecuentes.

Debido a que el contacto con las instrucciones y la ejecución de las mismas se realiza en distintos momentos, la capacidad para retener información juega un papel esencial en el seguimiento exitoso de las instrucciones (Allen y Waterman, 2015; Gathercole, Durling, Evans, Jeffcock y Stone, 2007; Yang, Gathercole y Allen, 2014). Una de sus principales restricciones es la capacidad de la MO para retener información esencial entre el momento en que las instrucciones fueron recibidas y el desempeño de la actividad (Gathercole et al., 2007).

De acuerdo con Yang (2011), la MO se encuentra altamente involucrada en el seguimiento de instrucciones (SI) y la central ejecutiva tiene el papel preponderante en ello, ya que se encuentra relacionada con el movimiento ocular para ayudar a retener la secuencia de acciones a ser realizada después de ser expuesto a la instrucción. Indica además, que el bucle fonológico únicamente desarrolla un papel secundario pues solo se encarga de mantener la información para prevenir su olvido a través de la repetición. Debido a que es cognitivamente demandante seguir una serie larga de instrucciones, Yang (2011) sugiere que se dividan las instrucciones en series menores o iguales a 5 , entre ejecuciones.

Existen diversos estudios que han identificado el rol de la memoria de trabajo en el seguimiento de instrucciones (Allen y Waterman, 2015; Jaroslawska et al., 2016). Además, se ha encontrado que las tareas concurrentes diseñadas para interferir con el control ejecutivo, el bucle fonológico y la agenda viso-espacial generan bajos desempeños en la habilidad para seguir instrucciones escritas (Yang et al., 2014). Estos resultados sugieren 
que las instrucciones verbales podrían almacenarse en el bucle fonológico y que podrían ser auxiliadas por información viso-espacial adicional en el ambiente, supeditados al control ejecutivo que retira información de las fuentes de almacenamiento.

Usualmente, las tareas diseñadas para estudiar la relación entre la capacidad de la memoria operativa (CMO) y el seguimiento de instrucciones, implican la ejecución de actividades inmediatamente después de leer múltiples instrucciones. En consecuencia, Jaroslawska et al. (2016) argumentan que es necesario verificar el tiempo transcurrido entre la recepción de una instrucción y su ejecución, puesto que cuando excede la duración de la MO, será necesario que el participante se mantenga repitiendo la información; lo cual podría requerir del apoyo de la memoria episódica de largo plazo, pero como su capacidad retentiva es menor a la CMO, podría ser menos efectiva para reproducir el contenido exacto. Ellos mismos sostienen que la agenda visoespacial no se encuentra relacionada con el SI; sino que, en estos casos son los aspectos verbales de la MO, los encargados de funciones sustantivas.

De acuerdo con Cowan, Chen y Rouder (2004), es posible retener en la memoria operativa un promedio 3.5 unidades de información. Al respecto, Ottem, Lian y Karlsen (2007) sostienen que el tamaño de dichas unidades varía y evoluciona en función de las habilidades lingüísticas de cada persona, pues ello implica la posibilidad de que se fusionen unidades, logrando así un volumen de información más amplio. Teniendo en cuenta lo anterior, es posible sostener que el desarrollo de la memoria operativa no se encuentra directamente relacionada con la edad cronológica, sino posiblemente con la experiencia de interacción lingüística con el ambiente. 
Por otro lado, se ha demostrado que la habilidad para recordar eventos pasados e imaginar eventos futuros decrece a medida que la edad avanza (Zavagnin, De Beni, Borella \& Carretti, 2016). Entre sus hallazgos, sostienen que la memoria operativa no presenta un deterioro significativo entre la adultez temprana y la avanzada. Sin embargo, argumentan que sí existen diferencias en términos de la imaginación y descripción de eventos futuros, lo cual podría tener efectos relacionados con el proceso de planificación para el desempeño de actividades específicas en el futuro $\mathrm{y}$, por tanto, en el seguimiento de instrucciones.

\section{Mediciones de la capacidad de la memoria operativa.}

Entre los instrumentos más usados actualmente, se encuentran las tareas complejas de alcance. Según Carruthers (2016), en ellas se maneja un distractor, tal como verificar si una frase es lógica o una operación es correcta, mientras se memoriza una lista de elementos no relacionados. $\mathrm{Y}$ aunque dichas tareas no son perfectas o puras, ya que las puntuaciones obtenidas están influenciadas por algo estable, con una pequeña contribución de error debido a fluctuaciones aleatorias, según Conway et al. (2005), una estrategia de investigación es administrar múltiples tareas porque una sola medida contiene la varianza específica de la misma y al recopilarse múltiples medidas, la varianza compartida es una representación más adecuada de la CMO. Además, ya que las correlaciones entre dichas tareas, generalmente van de 0.40 a 0.60 , sugieren que están aprovechando alguna capacidad común, pero también sugiere que no son iguales (Kane et al., 2004). A partir del estudio de Conway et al. (2005), diversos investigadores han aplicado medidas similares para validar sus adecuaciones y buscar asociaciones con diferentes constructos. 
Es interesante verificar en varios estudios, que es la tarea de Operaciones la más usual y en ocasiones la única medición de la $\mathrm{CMO}$, lo cual posiblemente se deba a lo establecido por Foster et al. (2015), quienes han encontrado que con la intención de minimizar el tiempo de medición, se usan pocos instrumentos para obtener conclusiones de la CMO. Por lo anterior y en línea con Conway et al. (2005), en el presente trabajo se ha decidido realizar mediciones con diferentes tareas de la CMO en los dominios visual y verbal, minimizando el tiempo al ser aplicadas de manera grupal y evitando que aquellos que son mejores en el procesamiento, aprovechen para ensayar los elementos memorizados. Las diferencias con otros trabajos, se centran básicamente en que cada prueba tiene 3 intentos en sus 4 niveles, que se distinguen por la cantidad de elementos a memorizar y que van desde 2 hasta 5; con un total de 42. Al aparecer el distractor, se debe elegir la respuesta correcta de entre dos opciones que aparecen en la pantalla. Cuando se recuperan los estímulos memorizados, el participante tiene que ingresarlos ya que no cuenta con opciones para elegir. No se tienen sesiones de práctica y en su lugar, los participantes pueden revisar videos demostrativos, previo a la resolución. Con el apoyo de un aplicador se pueden administrar, ya sea de manera individual o grupal, por lo cual se resuelven al ritmo del participante.

\section{METODOLOGÍA}

Desde un enfoque cuantitativo, se ha trabajado con un estudio de tipo correlacional, no experimental, transversal y prospectivo. A continuación, se presentan las características de la muestra, el procedimiento seguido, los instrumentos aplicados y las condiciones de aplicación de las pruebas. 


\section{Participantes.}

Se trabajó mediante un muestreo no probabilístico a conveniencia, con 33 universitarios de nuevo ingreso con una edad promedio de 18.5 años y con una proporción similar por género.

\section{Procedimiento.}

Memoria Operativa. Las mediciones de la CMO se realizaron en tres sesiones con duración de una hora y separadas por un día. En cada una, los participantes tuvieron acceso a una plataforma Moodle, donde se encontraban las pruebas y videos demostrativos de las mismas. El primer día realizaron las tareas de Lectura y Rotación, en el segundo, Operaciones y Simetría y en el tercero Razonamiento y Conteo, tratando de intercalar tareas de contenido verbal y visual. Se contó con el apoyo de los experimentadores y sus asistentes, quienes estuvieron presentes apoyando a los participantes. Previo a la resolución, luego de darles un tiempo para que revisaran los videos demostrativos, se les leían en voz alta las instrucciones del nivel 1 de cada prueba, mientras los alumnos las leían también en la pantalla de su computadora. Para reducir la distracción introducida por la presencia de otros participantes, se les instruyó para que iniciaran al unísono el nivel 1 de cada prueba y al término de los cuatro niveles, pararan y permanecieran sentados.

Seguimiento de instrucciones. La prueba (Solano-Uscanga, de Vries y Edel, 2018) se encuentra compuesta por 4 diferentes niveles de complejidad, presentados de manera aleatoria sin repetición y ejecutados en una sola sesión de forma grupal con una duración de hora y media. Cada nivel presenta secuencialmente 6 instrucciones para construir un modelo con bloques ajustables LEGO. La complejidad introducida consiste en solicitar la construcción de diseños desde dos dimensiones y con piezas de cualquier color (nivel 1) hasta diseños de tres dimensiones y con piezas 
de color específicos (nivel 4). En los niveles 1 y 3 se solicita el uso de piezas de cierto tamaño pero de cualquier color (genéricas), mientras que en 2 y 4, se pide de cierto color. Para realizar la prueba, se empleó una computadora por cada participante tanto para administrar las instrucciones como para construir los diseños. En la misma, el participante tenía acceso a una plataforma Moodle, con datos de acceso individuales y se encontraba ya operando, el software Lego Digital Designer ${ }^{\circledR}$ V4.3 con las piezas a emplear en cualquiera de los niveles. Cada nivel estaba integrado en una lección, en la cual no se podía revisar los pasos anteriores. Para la construcción de los modelos, el participante debía conmutar las pantallas de ambas aplicaciones.

Los participantes fueron evaluados previamente, debiendo cumplir con los requisitos:

I Diagnóstico negativo de Daltonismo

I Conocimiento lingüístico de las palabras "horizontal, vertical, izquierda, derecha y adyacente". Para ello, se requiere que el participante indique verbalmente su significado. En caso de que no conocieran alguna de las palabras, se les indicó su significado hasta comprobar la comprensión.

\Construcción exitosa de un modelo presentado visualmente (Figura $1)$.

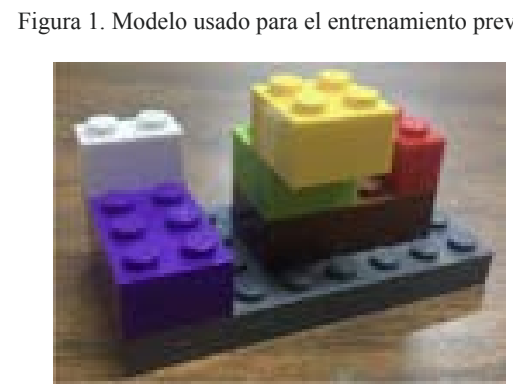

Fuente: Elaboración propia. 
Con respecto al último requisito, el diseño empleaba todas las formas de colocación, colores y cantidad de piezas empleadas a lo largo de la prueba y tuvo como propósito, entrenar al participante en el uso del software. Digno de mención, es que antes de iniciar cada nivel, el participante cargaba un archivo en la computadora con las piezas organizadas conforme a la figura 2, a manera de "hoja en blanco", y desde la cual construía el nivel de complejidad asignado. Una vez terminado éste, era necesario guardar el archivo en su formato nativo para posterior evaluación.

\section{Instrumentos}

Memoria Operativa. Se utilizó el software NeuronsWorkout, que consta de ocho tareas automatizadas, seis de ellas de tipo complejo y validadas en Esquivel-Gámez et al. (2018). De las seis, cuatro son usadas para el dominio verbal (lectura, operaciones, razonamiento y conteo) y 2 para el viso-espacial (rotación y de simetría). A continuación, se describen brevemente las citadas pruebas:

Figura 2. Piezas a ser usadas en cada nivel.

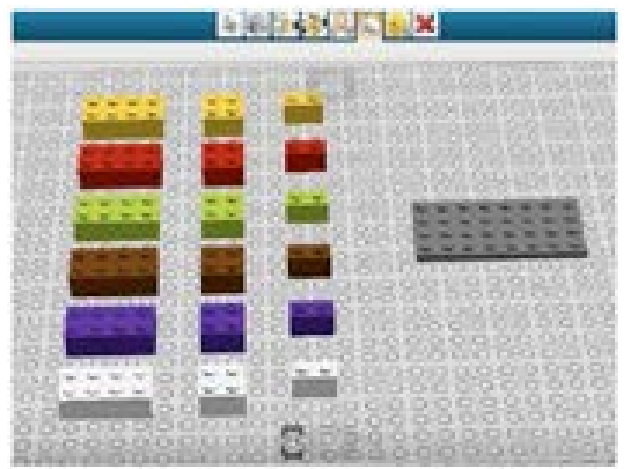

\begin{tabular}{lccc}
\multicolumn{4}{c}{ Plezas } \\
\hline \multicolumn{1}{c}{ Coler } & $\mathbf{1} \times \mathbf{2}$ & $\mathbf{2 \times 2}$ & $\mathbf{4 \times 2}$ \\
\hline Amanilo & 1 & 1 & 1 \\
Banco & 1 & 1 & 1 \\
Cafte & 1 & 1 & 1 \\
Morasto & 1 & 1 & 1 \\
Rojo & 1 & 1 & 1 \\
Vente & 1 & 1 & 1
\end{tabular}

Fuente: Elaboración propia. 
Alcance de lectura. Una serie de pares frase-letra aparecen y al tiempo que el participante indica si la frase es lógica o no (ej. El papel se mojó con la tierra cuando lo tiraron), debe memorizar la letra. Cuando termina la serie, se recuperan las letras conforme el orden de aparición.

Alcance de operaciones. Un grupo de pares palabra-operación aritmética aparece, para que el sujeto indique si la operación es correcta o no, al tiempo que memoriza la palabra asociada. Una vez que termina el grupo, las palabras memorizadas se ingresan en el orden en que aparecieron.

Alcance de conteo. Una serie de pares imagen-palabra aparece para que el participante memorice la cuenta de círculos azules e indique si dicha cuenta es un número par o no. Cada imagen contiene círculos y cuadrados azules más círculos verdes. Al final de cada serie, el participante debe registrar las cuentas, conforme al orden en que fueron presentados.

Alcance de razonamiento. Se muestra un conjunto de pares analogíapalabras y el sujeto debe elegir de las palabras, aquella que la complete correctamente. Después de resolver el conjunto, se recuperan las palabras de acuerdo al orden en que aparecieron.

Alcance de rotación. En una serie, aparece una letra en posición normal o girada, y luego de indicar Sí, o NO está rotada desaparece para luego exhibir una flecha que se debe memorizar. Al final de cada serie, se debe indicar el orden de presentación de cada flecha.

Alcance de simetría. En un grupo de pares de matrices, inicialmente aparece una con algunas celdas de negro, de modo que puede presentar simetría desde su eje vertical. El participante deberá marcar SÍ o NO es simétrica, para luego desaparecer y mostrar otra matriz con una celda roja 
y cuya posición debe memorizarse. Al final de cada grupo, en una matriz vacía se deben registrar, con números consecutivos el orden de aparición de la celda roja.

Seguimiento de Instrucciones. Al realizar esfuerzos por medir el seguimiento de instrucciones, diversos autores (Allison, Wilder, Lan, Flynn y Myers, 2011; Ndoro, Hanleym Tiger, Heal, 2006; Normand, Kestner y Jessel, 2010; Sy, Donaldson, Vollmer y Pizarro, 2014) definen el fenómeno como la ejecución exacta de una operación específica comunicada y no de una diferente. Teniendo en cuenta lo anterior, la medición del SI se realizó de manera manual, mediante un instrumento que guía el análisis de la ubicación, forma y color de las piezas empleadas en los modelos desarrollados por los participantes, en sus diferentes niveles de complejidad y validado por Solano-Uscanga y otros en 2018.

\section{RESULTADOS}

Una vez concluidas las mediciones, los datos fueron procesados con el software estadístico SPSS V15.0 y la información obtenida se describe enseguida.

Memoria Operativa. En cada nivel de prueba, se obtienen los datos: respuestas Correctas, respuestas en Orden, el tiempo promedio de reacción a cada planteamiento y el porcentaje de precisión ante la presencia del distractor. Para obtener los puntajes de las pruebas, en línea con Conway et al. (2005), se usó el procedimiento de puntuación de carga unitaria (PCU). Por cada prueba, los estadísticos descriptivos de tales puntajes se muestran en la tabla 1. Al realizar la revisión de la consistencia interna global, se encontró un valor de 0.904 para el Alfa de Cronbach, detectando que para el nivel 1 de todas las pruebas, el valor de la correlación elemento-total fue menor a 0.30 . De eliminarse dicho nivel, se logra que los coeficientes alfa 
superen el valor de 0.750 , excepto para el caso de Conteo, que obtiene un valor de 0.639 .

Seguimiento de instrucciones. Se evaluó cada modelo, agregando un punto por cada criterio ejecutado adecuadamente, de forma que la instrucción hipotética "coloca una pieza de 4 × 2 de color azul en la esquina inferior derecha...", en caso de que se haya ejecutado correctamente, se evaluaría sumando el tamaño de la pieza (1), el color de la misma (1), y luego su ubicación (1) para dar como resultado un puntaje de 3 en los niveles 2 y 4. En el nivel de complejidad más básico y en el tercero, el puntaje máximo por ítem es 2 , pues la instrucción no considera el color de las piezas empleadas. Los estadísticos descriptivos de las pruebas aplicadas, se muestran también en la tabla 1 .

A partir de la revisión del criterio de la distribución normal de los citados puntajes, se aplicaron las pruebas estadísticas correspondientes y los resultados se describen enseguida.

Memoria Operativa. Al comparar los promedios por tipo de dominio, se encontró que las de naturaleza verbal tuvieron una ventaja altamente significativa contra la del tipo viso-espacial (30 vs. 3 casos). Para realizar más comparaciones, se determinó dividir a los participantes conforme a dos niveles: Alto y Bajo. En el primero, se ubicaron aquellos cuyos puntajes eran superiores a la media en cuatro o más pruebas y en el segundo, a quienes los obtuvieron en tres o menos casos; de tal manera que el grupo Alto estuvo compuesto por 18 y el Bajo, por 15 participantes.

Seguimiento de instrucciones. Se detectó una diferencia significativa entre los puntajes a favor de las pruebas específicas contra las genéricas (21 vs. 9 casos). Para la prueba SI-2, se encontró una diferencia significativa 
a favor del grupo de CMO Alta (85.8 vs. $76.3, \mathrm{t}=-2.433, \mathrm{p}=0.021$ ). De manera análoga a las mediciones de la $\mathrm{CMO}$, se realizó una división entre niveles Alto y Bajo del rendimiento por prueba, tipo y global; conforme a la media grupal para cada caso, resultando para el último, un grupo Alto conformado de 22 y el Bajo de 11 integrantes.

Niveles de asociación. En la tabla 1 se presentan los niveles de correlación y significancia entre las diversas pruebas. En general, entre ambos dominios de la $\mathrm{MO}$, se encontró una correlación positiva altamente significativa de 0.604 . Al buscar asociaciones entre los puntajes de las pruebas y la edad, no se encontró alguna. En tanto, para las pruebas de seguimiento de instrucciones y las medias de los puntajes por dominio de la memoria operativa, se encontró que, para el caso del dominio verbal, se detectó una significativa de nivel menor con los niveles 2 y 3 ( 0.350 y 0.370 , respectivamente) y para el dominio viso-espacial, una de nivel bajo con las pruebas 2,3 y $4(0.362,0.373$ y 0.361$)$. Al obtener las medias de puntajes de SI, global, para pruebas genéricas y de color y las correspondientes a los dominios verbal y viso-espacial de las pruebas de $\mathrm{MO}$; se buscaron asociaciones a ese nivel, encontrando qué para ambos dominios, se encontró una similar significativa $(\mathrm{r}=0.385)$ con la media global del SI. También, se realizó un análisis por nivel de desempeño en las pruebas de MO (Alto versus Bajo), encontrando para los del nivel bajo, una de magnitud media $(r=0.622, p=0.013)$ entre la prueba de conteo y la prueba SI-3. Para el grupo de nivel alto, se encontraron una asociación negativa entre las pruebas de rotación y SI-4 $(\mathrm{r}=-0.566, \mathrm{p}=0.014)$ y una positiva entre la de simetría y SI-1 $(\mathrm{r}=0.652, \mathrm{p}=.003)$. 


\section{DISCUSIÓN DE RESULTADOS}

\section{Memoria Operativa}

Las menores medias se dan en las pruebas viso-espaciales, posiblemente debido a que las tareas visuales abarcan las habilidades menos maduras. Sin embargo, las tareas viso-espaciales son una medida valiosa de CMO, ya que pueden mejorar la sensibilidad de la mismas, en línea con Conway et al. (2005). Aunque menores a los obtenidos por Kane et al. (2004), quizás debido al tamaño de la muestra usada; los niveles de consistencia interna entre pruebas tuvieron diferencias similares. Dichos niveles fueron influidos por los puntajes del nivel 1, pudiendo indicar que quizás no tuvieron el entrenamiento suficiente, antes de iniciar las pruebas; sin embargo, el nivel de asociación entre las diferentes mediciones de la CMO va en línea con los resultados de Kane et al. (2004), excepto para la tarea de Razonamiento, cuyo nivel fue menor a 0.40 .

Tabla 1. Asociación entre puntajes de las pruebas.

\begin{tabular}{|c|c|c|c|c|c|c|c|c|c|c|}
\hline & 1 & 2 & 3 & 4 & 5 & 6 & 7 & 8 & 9 & 10 \\
\hline Lectura & 1 & $.687(* *)$ & $.654(* *)$ & $.537(* *)$ & $.605(* *)$ & $.695(* *)$ & .016 & .317 & .321 & .178 \\
\hline Rotación & & 1 & $.620(* *)$ & $.601(* *)$ & $.356(*)$ & $.627(* *)$ & .017 & .192 & .308 & .215 \\
\hline Operaciones & & & 1 & $.585(* *)$ & $.626(* *)$ & $.548(* *)$ & .116 & $.370(*)$ & $.419\left(^{*}\right)$ & $.375\left(^{*}\right)$ \\
\hline Simetría & & & & 1 & $.493(* *)$ & $.479(* *)$ & $.364(*)$ & $.396(*)$ & .306 & $.405\left(^{*}\right)$ \\
\hline Razonamiento & & & & & 1 & $.383\left({ }^{*}\right)$ & .105 & $.405\left(^{*}\right)$ & .242 & .335 \\
\hline Conteo & & & & & & 1 & .218 & $.345\left(^{*}\right)$ & $.369\left(^{*}\right)$ & .194 \\
\hline SI-1 & & & & & & & 1 & $.579(* *)$ & .243 & $.395\left(^{*}\right)$ \\
\hline SI-2 & & & & & & & & 1 & .199 & $.574(* *)$ \\
\hline SI-3 & & & & & & & & & 1 & $.413\left({ }^{*}\right)$ \\
\hline SI-4 & & & & & & & & & & 1 \\
\hline Media & 0.89 & 0.65 & 0.89 & 0.75 & 0.77 & 0.91 & 85.02 & 72.73 & 81.48 & 91.92 \\
\hline Desv. Típica & 0.114 & 0.226 & 0.09 & 0.137 & 0.125 & 0.096 & 11.29 & 19.684 & 12.001 & 15.658 \\
\hline
\end{tabular}

$* \mathrm{p}<.05, * * \mathrm{p}<.01$. Fuente: Elaboración propia 


\section{Seguimiento de Instrucciones}

La media global lograda pudo deberse, entre otros factores favorecedores, a que: el tiempo transcurrido entre la lectura de la instrucción y su ejecución era mínimo, conforme a Jaroslawska et al. (2016); la cantidad de instrucciones por nivel fue de 6, similar a lo propuesto por Yang (2011) y finalmente, la demanda de habilidad es psicomotoras en la construcción era menor (virtual en lugar de física). Es interesante observar que, aun cuando los participantes construyeron los modelos en distinto orden, los puntajes promedio fueron en aumento conforme crecía la dificultad, opuesto a lo encontrado en los distintos niveles por prueba de MO, en cuyo caso conforme aumentaba el nivel, disminuía el promedio. Al parecer, en el caso del SI, es posible que la presencia de más elementos en una instrucción, active procesos atencionales que favorezcan el seguimiento de instrucciones, ya que la exigencia de cierto color y manejo de tres dimensiones, introdujo elementos de mayor especificidad y por tanto, de apoyo.

\section{Asociaciones entre MO y SI.}

Al detectarse que para ambos dominios de la $\mathrm{MO}$, los niveles asociativos con el puntaje general del SI fueron casi similares $(r=0.385)$, se puede determinar que al seguir instrucciones, la central ejecutiva integra estímulos de los almacenes fonológico y viso-espacial, conforme a Yang et al. (2014). En línea con ellos, es posible determinar que la CMO está relacionada con la capacidad de codificar secuencias verbales significativas, al aprovechar el almacenamiento fonológico y además, utilizar pistas viso-espaciales que el tipo de tarea impuso, para facilitar el rendimiento de la memoria. Sin embargo, en el caso del dominio viso-espacial, el nivel de significancia de la correlación con la capacidad de seguir instrucciones, no se alinea con lo encontrado por Jaroslawska et al. (2016), quizás por el tipo de mediciones de la $\mathrm{CMO}$ y de las tareas de seguimiento de instrucciones que ellos usaron. 
Por nivel de CMO, la menor media y mayor desviación en los puntajes se obtuvo en la viso-espacial, Alcance de Rotación, lo cual quizás explique la asociación negativa con el nivel 4 de la prueba SI (Color específico y Tridimensional), para el grupo de $\mathrm{CMO}$ alto. Para este mismo grupo, posiblemente debido a que la prueba de Alcance de Simetría maneja matrices, se asoció positivamente con el manejo de un modelo bidimensional del nivel 1. En cambio, para el grupo CMO Bajo, el procesamiento de un patrón visual en la prueba de Conteo pudo haber contribuido al mejor desempeño en el nivel bidimensional genérico.

La distribución de participantes por nivel de la CMO y del SI, indica una proporción menor de participantes en CMO alta que en el alto nivel de SI (55\% vs 67\%); lo cual puede deberse al aumento progresivo del puntaje promedio conforme aumentaba el nivel.

\section{CONCLUSIONES}

Para el presente estudio, es posible concluir que los puntajes de seguimiento de instrucciones aumentan a medida que la complejidad de la tarea se eleva. Sin embargo, es posible que tal comportamiento cambie con el aumento excesivo de la complejidad instruccional. Por ello, se plantea la necesidad de realizar arreglos experimentales con gamas de complejidad más amplias a la presentada.

Conforme a los elementos distintivos de los instrumentos usados, se puede concluir que existe una asociación significativa positiva entre la habilidad para seguir instrucciones y la capacidad de la memoria de trabajo, tanto en el dominio verbal como en el viso-espacial.

Debido a la naturaleza del contexto en el que se encontraron los participantes, su grupo etario es prácticamente el mismo, hecho que podría 
explicar la ausencia de diferencias al analizar la edad y los puntajes en las distintas pruebas realizadas, por lo cual es necesario realizar estudios con más grupos etarios con el fin de aclarar esta situación.

Una línea de trabajo futuro es determinar el efecto de la Memoria Operativa, al implementar ensayos en los cuales se introduzcan distractores, al tiempo que los participantes se apropian de las instrucciones por lectura, imaginación, visualización de la acción o ejecución de la misma.

En este trabajo, se ha demostrado que es posible implementar ambientes virtuales que permitan experimentar con el seguimiento de instrucciones de manera controlada y a la vez medir la capacidad de la memoria operativa, para determinar el impacto de su correlación, en ambientes de interacción convencional. Al mejorarse las condiciones bajo las cuales se hacen las mediciones, se pueden proporcionar evaluaciones ecológicamente válidas y útiles, para los profesionales psico-educativos interesados en detectar aquellos alumnos con riesgo de un progreso académico lento. Aprovechando el bajo costo que representa su implementación, es viable reproducir esta iniciativa en diversos contextos educativos para, al tiempo de mejorar los procedimientos de medición, determinar con mayor certeza los hallazgos. También, es posible agregar al ambiente virtual, tareas de entrenamiento adaptativo de la memoria de trabajo con actividades que se asemejen más a los usos diarios de la memoria operativa y del seguimiento de instrucciones.

\section{REFERENCIAS BIBLIOGRÁFICAS}

I Allen, R., y Waterman, A. (2015). How does enactment affect the ability to follow instructions in working memory? Memory and cognition, (43), 555-561. Disponible en https://www.ncbi.nlm.nih.gov/ pubmed $/ 25377510$

I Allison, J., Wilder, D., Teo, H., Flynn, A., y Myers, K. (2011). The effect of the availability of a similar toy on compliance with instructions to surrender a toy among preschool children. Behavioral Interventions, (26), 167-178. 
I Arteaga, G. y Pimienta, H. (2006). "Memoria operativa y circuitos corticales", Revista de la Facultad de Medicina Universidad Nacional Colombia, vol. 54, núm. 4, pp. 248-268. Disponible en: DOI: 10.15446/ revfacmed

I Baddeley, A. (2012). Working memory: theories, models, and controversies. Annual Review of Psychology, 63, 1-29. DOI: http://doi.org/10.1146/annurev-psych-120710-100422.

I Baddeley, A. y Hitch, G. (1974). Working memory. The psychology of learning and motivation, (8), 47-90. DOI: 10.1016/S0079-7421(08)60452-1

I Carruthers, P. (2016). La evolución de la memoria de trabajo, Ludus Vitalis, (21) 40, 99-124. Disponible en https://www.centrolombardo.edu.mx/ludus-vitalis/la-maquinaria-mental-humana-num-40-2013/laevolucion-de-la-memoria-de-trabajo-ludus-vitalis/

I Conway, A. R., Kane, M. J., Bunting, M. F., Hambrick, D. Z., Wilhelm, O., y Engle, R. W. (2005). Working memory span tasks: A methodological review and user's guide. Psychonomic Bulletin Review, 12, 769 786. Disponible en https://link.springer.com/article/10.3758/BF03196772

I Cowan, N., Chen, Z., Rouder, J. (2004). Constant capacity in an immediate serial-recall task: A logical sequel to Miller (1956). Psychological Science, 15, 634-640.

I Donadelli, J. y Strapasson, B. Effects of Monitoring and Social Reprimands on Instruction-Following in Undergraduate Students. Psychological Record, n. 65, p. 177- 188, 2015.

I Esquivel-Gámez, I., Martínez-Olvera, W., Galvez-Buenfil, K.E., Barrios-Martínez, F.L., López-Azamar, B., Córdoba-Del Valle, R., y Medina-Cruz, H. (2018). NeuronsWorkOut: Experiencias en su aplicación para medir la capacidad de la memoria operativa. En I. Esquivel-Gámez, R. Edel-Navarro, G. AguirreAguilar y J. Balderrama. (Eds.), Memoria operativa: Medición y propuesta para su desarrollo, apoyadas en TIC. Ciudad de México: Porrua Print, pp. 69-94.

$\checkmark$ Etchepareborda, M.C. y Abad-Mas, L. (2005). "Memoria de trabajo en los procesos básicos del aprendizaje", Revista de Neurología, vol. 40, núm. 1, pp. 79-83.

I Flores, J. y Ostrosky-Shejet, F. (2012). Desarrollo neuropsicológico de lóbulos frontales y funciones ejecutivas, Ciudad de México: Manual Moderno.

I Foster, J. L., Shipstead, Z., Harrison, T. L., Hicks, K. L., Redick, T. S., y Engle, R. W. (2015). Shortened complex span tasks can reliably measure working memory capacity. Memory and cognition, 43(2), 226236. Disponible en https://link.springer.com/article/10.3758/s13421-014-0461-7

1 Ganier, F., y de Vries, P. (2016). Are instructions in video format always better than photographs when learning manual techniques? The case of learning how to do sutures. Learning and instruction, (44), 8796. Disponible en https://www.sciencedirect.com/science/article/pii/S095947521630024X

I Gathercole, S., Durling, E., Evans, M., Jeffcock, S., y Stone, S. (2007). Working memory abilities and children's performance in laboratory analogues of classroom activities. Applied Cognitive Psychology, 22, 1019-1037. Disponible en https://onlinelibrary.wiley.com/doi/abs/10.1002/acp.1407

I Jaroslawska, A., Gathercole, S., Logie, M., y Holmes, J. (2016). Following instructions in a virtual school: Does working memory play a role? Memory and cognition, (40), 580-589. DOI: 10.3758/s13421-015$0579-2$ 
I Jonides, J., Lacey, S. C. y Nee, D. E. (2005). "Processes of working memory in mind and brain", en Current Directions in Psychological Science, vol. 14, núm. 1, pp. 2-5. Disponible en: DOI:10.1111/j.09637214.2005.00323.x

I Kane, M. J., Hambrick, D. Z., Tuholski, S. W., Wilhelm, O., Payne, T. W., \& Engle, R. W. (2004). The generality of working memory capacity: a latent-variable approach to verbal and visuospatial memory span and reasoning. Journal of Experimental Psychology: General, 133(2), 189.

I Miyake, A.; Friedman, N. P.; Emerson, M. J.; Witzki, A. H.; Howerter, A. y Wager, T. D. (2000). "The unity and diversity of executive functions and their contributions to complex "frontal lobe" tasks: A latent variable analysis", Cognitive psychology, vol. 41, núm. 1, pp. 49-100. Disponible en: DOI:10.1006/ cogp.1999.0734.

I Ndoro, V., Hanley, G., Tiger, J., y Heal, N. (2006). A descriptive assessment of instruction-based interaction in the preschool classroom. Journal of applied behavior analysis, 39(1), 79-90. Disponible en https:// www.ncbi.nlm.nih.gov/pubmed/16602387

I Normand, M., Kestner, K., y Jessel, J. (2010). An analysis of stimuli that influence compliance during the high-probability instruction sequence. Journal of Behavioral Education, 43(4), 735-738. Disponible en https://www.ncbi.nlm.nih.gov/pubmed/21541158

1 Ottem, E., Lian, A., \& Karlsen, P. (2007). Reasons for the growth of traditional memory span across age. European Journal of Cognitive Psychology, 19(2), 233-270. DOI: https://doi. org/10.1080/09541440600684653

I Saeed, T. (2011). A Comparative Study of Working Memory in Children with Neurodevelopmental Disorders. (Tesis Doctoral). National University of Ireland Maynooth, Irlanda

I Solano-Uscanga, E., de Vries, W., y Edel, R. (2018). La contribución didáctica de las instrucciones en la educación virtual. Tesis doctoral. Benemérita Universidad Autónoma de Puebla.

I Sy, J., Donaldson, J., Vollmer, T., y Pizarro, E. (2014). An evaluation of factors that influence children 's instruction following. Journal of applied behavior analysis, 14(1), 101-112. Disponible en https:// onlinelibrary.wiley.com/doi/pdf/10.1002/jaba.94

I Yang, T. (2011). The role of working memory in following instructions. Tesis doctoral. University of New York.

I Yang, T., Gathercole, S., y Allen, R. (2014). Benefit of enactment over oral repetition of verbal instructions does not require additional working memory during encoding. Psychonomic Bulletin Review, 21, 186-192. Disponible en https://www.ncbi.nlm.nih.gov/pubmed/23817922

I Zavagnin, M., De Beni, R., Borella, E., \& Carretti, B. (febrero, 2016). Episodic future thinking: the role of working memory and inhibition on age-related differences. Aging clinical and experimental research, 28(1). 109-119. 
Envío a dictamen: 25 de mayo de 2020

Reenvío: 16 de junio de 2020

Aprobación: 7 de Julio de 2020

Ismael Esquivel Gámez. Doctor en tecnología de información y análisis de decisiones. En el campo profesional, se ha desarrollado en el ámbito de las TIC, como desarrollador, consultor, empresario e investigador. Actualmente, se desempeña como profesor de tiempo completo en las áreas de Multimedia Educativa y Modelos y ambientes educativos de la Universidad Veracruzana, Región Veracruz. Correo electrónico: iesquivel@uv.mx.

Eric Efraín Solano Uscanga. Licenciado el Psicología, Maestro en Investigación en Psicología aplicada a la Educación y Doctor en Sistemas y Ambientes Educativos. Certificado por Apple, Google y Microsoft en docencia mediada por tecnología. Fellow de la cátedra UNESCO-ICDE Movimiento Educativo Abierto 2019. Se desempeña como profesor en la Universidad Veracruzana, así como docente y director de Educación en línea de la Universidad de Xalapa. Correo electrónico: efrain@ux.edu.mx 